\title{
SÉMIOTIQUE ET POLITIQUE : NARRATIVITÉ ET TRANSFORMATION
}

\begin{abstract}
Juan Alonso $^{1}$
La prolifération du narratif et du concept de récit lui-même a été amplement constatée dans le discours et dans la communication politiques et médiatiques. Pour autant cela n'a pas toujours été accompagné d'une augmentation des études sur le statut théorique de ce narratif omniprésent. Cet article étudie la communication et le discours politiques à la lumière de la notion sémiotique de narrativité, conçue comme théorie du sens des processus et des formes de l'action et de la transformation du discours, et essaie de penser une sémiotique du politique à partir de l'analyse approfondie du concept de transformation et de ses différentes modalités d'existence et de ses régimes de fonctionnement.
\end{abstract}

\section{Le récit dans le discours politique}

Il y a quelque chose de surprenant dans l'histoire de la sémiotique (et conséquemment dans sa place et dans sa pratique au sein des études politiques et des discours sociaux) : le paradoxe du succès de certains de ses notions et outils en dehors de la discipline coïncidant en même temps avec l'abandon par les sémioticiens eux-mêmes de ces mêmes outils et concepts, comme si l'arrivée de nouvelles problématiques et

1 Juan Alonso est Maître de conférences à l'Université Paris-Descartes.

Recherches en communication, $\mathrm{n}^{\circ} 41$ (2014). 
de nouveaux concepts dans la discipline l'obligeait à laisser de côté les anciens acquis de la théorie. Soit qu'elle abandonne la continuité de l'exploration théorique autour d'un concept ou d'un pan de la théorie car on considère que le tour de la question a déjà été fait - soit qu'elle n'explore plus cette dimension dans les analyses concrètes des discours ou des pratiques signifiantes qu' elle se donne comme objet-, puisqu'elle s'occupe désormais d'autres dimensions du discours qui semblent plus pertinentes et enrichissantes pour le cours général de la discipline - la sémiotique semble parfois laisser à d'autres disciplines le soin d'utiliser ou de manipuler certains de ses concepts. Pour ces disciplines - comme le marketing, politique ou commercial -, la sémiotique n'est qu'une sorte de boîte à outils dans les meilleurs des cas ou tout simplement le fournisseur de quelques concepts de schématisation simplificateurs ou de notions passe-partout qui se répandent avec une plus ou moins grande pertinence et efficacité, comme c'est le cas pour ceux qui constituent l'objet de cet article, à savoir, les concepts de récit et de narrativité.

En effet, la prolifération de ces deux notions dans différents champs disciplinaires va de pair curieusement avec sa raréfaction dans le champ sémiotique, disparition sur laquelle je reviendrai et dont je donnerai quelques exemples, quelques explications et quelques tentatives de réactivation justement à l'aide de ces nouveaux concepts plus en vogue ces dernières années en sémiotique. Ainsi donc les objectifs de ce travail sont doubles : d'un côté, revoir l'utilité de la grammaire sémiotique narrative dans l'étude des discours politiques en introduisant le concept de mode d'existence et à l'aide des nouveaux outils développés par la sémiotique tensive, et, de l'autre côté, repenser la notion de transformation en sémiotique à la lumière de ces nouveaux outils de la théorie.

Mais pour l'instant j'aimerais commencer par constater la prolifération, le succès - si l'on peut tenir pour tel pour une discipline scientifique la «popularité » voire la « peopolisation » de ses notions des concepts de récit et de narrativité. Cette diffusion a atteint un tel déploiement, une telle généralisation et, parfois, une telle banalisation qu'on n'est plus étonné d'entendre dans la bouche d'un haut dirigeant du parti au pouvoir en Espagne des phrases du type : «Il manque un récit au gouvernement " pour expliquer la désaffection des citoyens espagnols pour les hommes et femmes politiques et pour la gestion que ceux-ci font de la chose publique ; ou encore l'affirmation d'un analyste économique qui essayait d'expliquer les raisons de l'échec de la politique de l'ancien président du gouvernement espagnol avec des 
affirmations comme : «Le président du gouvernement n'a pas trouvé le récit pour expliquer la crise ».

Cette prolifération et du récit et du concept lui-même a été amplement constatée dans les domaines politique et médiatique. Cela ne signifie pas pour autant une augmentation des études du narratif dans d'autres ordres de discours différents - politiques, historiques, médiatiques, scientifiques, etc. - par la sémiotique mais plutôt le contraire. Force est de constater aujourd'hui que les études narratives se développent plus amplement dans d'autres disciplines qu'au sein de celle qui les a inventées et développées. C'est ainsi que, depuis très longtemps, la réflexion sur la place de la narrativité dans la construction du sens de l'histoire constitue un des angles de recherche essentiels de l'historiographie récente, où le débat tourne souvent autour des régimes de vérité, de fiction ou de fausseté - et de leurs marqueurs discursifs, par exemple à travers les différentes stratégies énonciatives d'objectivisation ou de subjectivisation des faits - qui définissent les récits historiques.

Cette perspective est très différente de celle des travaux critiques qui se contentent de dénoncer une soi-disant utilisation manipulatrice du récit à des fins politiques, économiques ou commerciales, comme c'est le cas dans les travaux critiques sur le storytelling. Cette perspective critique confond très souvent l'utilisation du récit à des fins mystificatrices, les structures narratives sous-jacentes au discours politique et médiatique elles-mêmes, et la sémiotique narrative comme méthodologie d'analyse du récit et du discours.

On ne comprend pas trop bien si la question analysée par ces dernières études sur la place du récit dans la communication politique est celle du récit comme objet ou celle de la théorie du récit en soi. Dans cette confusion des niveaux de pertinence de l'analyse on ne sait pas trop bien si ce qu'on met en cause, c'est la sémiotique-objet (c'est-àdire le récit politique et ses usages en vue de ce que Christian Salmon appelle « la fabrique à formater les esprits » (Salmon, 2007)) ou la sémiotique comme métalangage, c'est-à-dire la sémiotique narrative justement comme méthodologie d'analyse de ces stratégies narratives qui apparemment envahissent le discours politique et social.

Or comment rendre compte du fonctionnement de ces mêmes récits à usage « manipulateur » si, en critiquant cet usage des récits, on condamne et on bannit en même temps les outils à même d'expliquer leurs formes et leurs mécanismes ? Par ailleurs, cette notion de « récit » telle qu'elle est citée et utilisée tient plus du passe-partout conceptuel 
que d'une véritable notion explicative. Elle ne distingue pas en fait un niveau d'analyse d'un autre et l'étude de ces nouvelles formes de la construction et de la manipulation de l'opinion publique demeure ainsi une simple dénonciation vague du marketing généralisé dans une grande partie du discours politique et social dominant. Sans cette distinction entre niveaux de pertinence dans l'étude de ces discours, l'analyse demeure purement démonstrative et illustrative d'une hypothèse avec laquelle on peut être d'accord mais qui n'explique ni les mécanismes du discours ni les effets de ceux-ci, par exemple dans la construction de ce qui aurait été l'emprise du marketing sur les campagnes de Nicolas Sarkozy et de Ségolène Royal lors de la campagne présidentielle de 2007. Il ne s'agit donc pas de dire si le récit s'est ou non emparé du discours politique mais d'indiquer de quel type de récit il s'agit et d'en dégager les structures et les règles ainsi que les régimes qui les organisent.

Cette absence d'une différenciation des niveaux d'analyse et d'organisation de la narrativité a en fait pour origine l'absence même d'une interrogation sur la notion de récit. On se limite à affirmer que celui-ci a colonisé le discours et la pratique politiques. De la même manière que Paul Ricœur (1983, pp. 365-384) montrait dans son analyse de La Méditerranée de Braudel que l'introduction du paradigme de la « longue durée » en histoire provoquait non pas la disparition du récit dans l'histoire mais l'apparition d'un autre type de récit (une « quasiintrigue ", dit Ricœur) fondé sur l'unité née des interférences des trois types de temporalité différents dont parle Braudel (temporalités structurelles, cycliques et événementielles), il faudrait dans les études $\mathrm{du}$ fonctionnement du récit dans le politique mettre en évidence aussi, entre autres, les différents régimes de temporalité qui y sont mis en fonctionnement. Un bon cas et exemple pour ce genre d'analyse serait l'étude du fonctionnement de ces trois temporalités dans la narration sur la crise économique sans se limiter à affirmer que l'économie est entrée dans une sorte de «fictionnalisation ». Dans ce sens, nous aurions intérêt, par exemple dans l'analyse du discours économique et politique sur la crise actuelle, à rendre compte de sa « mise en intrigue » à travers l'étude des effets d'interférences, d'écarts ou de concurrences entre les différentes modalités du temps, pour reprendre l'idée de Ricœur sur Braudel. Ainsi, on retrouverait dans le discours économique et politique un récit pour rendre compte de la crise de l'économie grecque construit de la manière suivante : des événements (la découverte du déficit abyssal de l'économie grecque, par exemple, avec ses effets d'intensification 
dramatique et passionnelle) jalonnent et rythment un cycle et l'orientent dans un sens ou dans un autre (alors on raconte l'inexorable décadence et la supercherie de l'économie hellénique depuis quelques années) avant de l'intégrer dans une temporalité plus étendue, une structure culturelle et sociale (et alors on affirme la fragilité endémique des institutions ou la corruption structurelle des pays du sud).

\section{Sémiotique politique et narrativité}

Si d'un côté donc il y a eu une très grande présence du récit et de la narrativité en dehors de la sémiotique, avec une conceptualisation assez confuse et vague, nous essayerons de comprendre pourquoi dans une grande mesure la sémiotique qui travaille sur les discours et les pratiques sociaux et politiques a abandonné la perspective sémionarrative comme le lieu des développements de ses dernières avancées théoriques et aussi comme lieux et cas des discours à explorer. Plusieurs critiques du modèle de la syntaxe narrative ont été faites à l'intérieur même de la sémiotique, surtout de la part de la socio-sémiotique (Landowski, 2004), considérant ce modèle comme trop rigide puisqu'il ne conçoit l'action humaine et son sens qu'à partir d'une économie d'échange d'objets entre sujets avec des opérations de conjonction et de disjonction (donc de possession ou de séparation). Ces critiques ont contribué dans une certaine mesure à un abandon de la sémiotique du narratif alors que ce qui était en question, c'était un régime de transformation narrative particulier et pas tellement la transformation en soi, ce qui continue à être le cœur même de la signification et du politique, vu que l'objet principal de celui-ci est la transformation des sujets, des états et des choses publiques et collectives. Dans ce sens, la sémiotique du politique doit être d'abord, mais évidemment pas en exclusivité, une théorie des processus et des formes de l'action et de la transformation, ce qui a été depuis ses origines le but du modèle narratif de la sémiotique, pour lequel justement le sens n'est saisissable que dans ses métamorphoses : d'un état de popularité à un autre de désaveu, de la possession du pouvoir à sa perte, d'une situation de conflit et désaccord à une action commune, de la soumission à la révolte, etc. Cela ne fait aucun doute que ce travail n'est pas le seul à réaliser par une sémiotique du politique - il y a, bien entendu, des pans entiers du politique qui ne sont pas inclus dans ce programme, comme l'essentielle dimension passionnelle, par exemple, mais repenser les modes et les régimes de transformation sous forme narrative est un travail nécessaire 
à la vue des définitions plus qu'ambiguës et imprécises qui définissent aujourd'hui le concept de narratif et de récit dans le discours ambiant sur le politique.

Il faudrait bien entendu élargir cette notion de transformation un peu trop schématique des premiers modèles de la sémiotique narrative, avec un passage d'un état de disjonction à un état de conjonction et vice versa, pour expliquer les phénomènes politiques où les transformations ne sont pas aussi nettes que ce que ce modèle prévoyait. Ainsi, il ne s'agirait pas uniquement de rendre compte de la perte ou de la prise du pouvoir dans une logique causale implicative et prévisible, avec ce que ces deux actions ont de changement catégorique, mais aussi de décrire par exemple l'érosion continue de l'autorité, la déformation ou le glissement silencieux et discret d'une position politique vers une autre ou au contraire les sursauts inespérés, les retournements bruyants et brutaux du transformisme politique et des trahisons idéologiques ou encore les acharnements inexplicables dans des pratiques et des discours déjà extrêmes. Par exemple, il serait très intéressant d'explorer le régime syntagmatique concessif hyperbolique (Zilberberg, 2004), pour reprendre le concept proposé par Claude Zilberberg, pour comprendre la logique qui se trouve derrière un certain discours politico-économique qui consiste à faire couler ce qui est déjà enfoncé avec une obstination sans faille ${ }^{1}$.

Le travail d'une sémiotique du politique doit être donc le déchiffrage de cette boîte noire qui est le passage d'un état socio-politique à un autre ou celui de sa simple modification. La sémiotique du politique doit pouvoir rendre compte par exemple de la transformation d'un discours de révolte ou de contestation en un discours révolutionnaire ou insurrectionnel, en repérant les variations tensives qui gèrent le syntagme passionnel menant à une action révolutionnaire ; elle doit pouvoir expliquer aussi les modulations syntagmatiques du changement opéré à l'intérieur d'une pratique ou d'un discours sur la liberté et la sécurité à travers l'analyse des infléchissements modaux concernant un comportement social avec un basculement d'un discours défini par la

1 Dans Le Monde daté du 18 mai 2013, l'économiste Jean-Luc Gréau affirmait à propos des visions et des mesures économiques mises en place ces dernières années : "On ne sait pas panser les plaies. Aujourd'hui, on traite le mal par le mal ». Cela ne fait aucun doute que cette " médecine » économique est de l'ordre du concessif hyperbolique où l'on « ouvre le béant », pour reprendre les catégories sémantiques utilisées par Claude Zilberbeg pour rendre compte de ce type de régime syntagmatique. 
modalisation du " possible » à un autre marqué par une modalisation du type " nécessaire ». La sémiotique narrative aurait tout intérêt à aborder les stratégies de discours mises en œuvre pour l'abandon ou les modifications des contenus des promesses politiques et les stratégies d'intégration et d'articulation des différentes temporalités dans le management des modifications de ces mêmes promesses. Elle devrait également être en mesure de donner une explication non causale et immanente aux discours et aux pratiques sociales des phénomènes d'accélération et de décélération techniques et sociales à partir, par exemple, du concept de " forme de vie » (pour étudier par exemple d'un point de vue sémiotique des pratiques comme le "speed dating » ou, dans le registre inverse, le "slow food » (Marrone, 2010)), ou le fonctionnement des régimes de tempo (pour l'analyse des questions liées à l'innovation sociale) comme socle pour comprendre le sens de ce qui constitue un des objets majeurs de la sociologie actuelle (Rosa, 2010).

\section{Régimes de la transformation}

Ainsi donc une sémiotique du politique sera d'abord une théorie des transformations et des régimes ainsi que des modalités de celles-ci. L'adoption de la notion de transformation (qui n'est pas dans l'absolu nouvelle en sémiotique) permet d'inclure tous les types de mouvements qui s'opèrent dans la pratique et dans le discours politique, aussi bien ceux qui sont le produit des programmes et des actions donnant lieu à des renversements programmés, affichés et complets, et donc accomplis dans leur totalité, ainsi qu'aux autres types de modifications et de mutations qui se réalisent sans qu'un programme ou une modalisation particulière en soit à l'origine, qui ne s'affichent pas et ne se revendiquent pas, et surtout qui ne sont pas nécessairement complètement réalisés et qui restent en cours de réalisation.

La transformation donc peut être alors perçue de deux points de vue différents :

a) en tant qu'action qui vise à changer un état des choses

et

b) en tant que résultat ou produit de cette action 
Ainsi donc la première distinction à introduire dans une sémiotique de la transformation appliquée au politique concerne les formes aspectuelles que celle-là peut revêtir.

La première de ces formes, celle de l'acte en tant que tel, est caractérisée d'un point de vue aspectuel par l'imperfectif, car en tant qu'acte elle n'est appréhensible qu'en cours d'action, jamais dans son achèvement. Les aboutissements et les contours politiques de ce type de configuration de la transformation peuvent se révéler extrêmement complexes et subtils et se manifester à différents niveaux du discours :

a) au niveau de la temporalité avec la production par exemple des effets de retardement, de précipitation, d'anachronisme, de désynchronisation, etc. En résumé, toutes les formes qui empêchent l'achèvement, qui entravent - soit par dilation ou par désajustement - l'accomplissement de la transformation. Un exemple de ce type d'imperfectivité de la transformation dans le politique est l'intégration de celle-ci à l'intérieur d'une programmation stratégique, un peu comme dans la construction d'une recette de cuisine ou d'un protocole quelconque, où les actions s'intègrent hiérarchiquement les unes dans les autres et exigent un ordre de réalisation : alors, de la même manière que pour réaliser une omelette il faut d'entrée battre les œufs, on prétendra que la réalisation d'un certain engagement politique pris lors d'une campagne électorale nécessite auparavant l'accomplissement d'autres mesures préalables qui seront présentées comme faisant partie déjà du projet initial, lequel ne sera jamais invoqué comme complètement réalisé. Cette forme aspectuelle établit donc une segmentation de l'action politique en générant des seuils entre segments ordonnés (entre les " précédents » toujours invoqués en politique (la situation laissée par l'adversaire par exemple), en « désignant» les « étapes nécessaires à respecter et à suivre » («d'abord ceci, ensuite cela ») et par conséquent en créant un effet de progressivité de l'action.)

b) au niveau de la spatialité, cette imperfectivité de la transformation en politique se donne comme le produit d'une segmentarisation de l'espace d'application de l'action, dans une sorte d'opération rhétorique d'expansion à travers une synecdoque d'étendue : quelque chose comme une 
expérimentation qui a lieu dans un cadre délimité, afin, dit-on, de tester une mesure. On n'abandonne pas la transformation, on ne l'accomplit pas complétement, il en reste une partie qui joue le rôle du lieu témoin et de la trace de sa continuité. Si l'espace de la réalisation d'un programme narratif, d'un projet quelconque en politique, a lieu dans ce qu'on peut appeler un espace «topique », ce type de stratégie de localisation de la transformation construit un nouvel espace, un espace «paratopique », c'est-à-dire une sorte d'espace « incubateur », comme on dit des espaces dédiés aux entreprises naissantes et pas tout à fait bien installées dans le paysage industriel. De cette manière, l'agir et le discours politiques placent l'action de transformation dans un état et dans un lieu de " mise en préparation » et de " mise à l'épreuve », c'est-à-dire un espace où l'on est en train d'acquérir les compétences nécessaires pour plus tard réaliser la performance en question.

c) au niveau du mode d'existence, car cette " mise à l'épreuve » de l'action moyennant une localisation particulière de celleci prend également la forme d'un type particulier de mode d'existence de l'action politique. Au fond, la figure rhétorique de la synecdoque évoquée à l'instant convoque aussi une lecture en termes de modes de présence, comme s'il s'agissait de présenter l'action et le discours à deux niveaux de « réalité différents » : d'un côté une partie " réalisée », présente, et de l'autre, une partie supposée potentialisée (le tout soidisant contenu dans la partie). L'action n'est pas jugée par sa réalisation mais par sa virtualité, par les contenus virtuels qu'elle contient. On l'aura remarqué, c'est le «Attendez, vous verrez les résultats » ou la célèbre « boîte à outils » que le gouvernement de François Hollande avait mise en application pour sortir de la crise et qui apparemment contenait déjà virtuellement les solutions. Le discours et les actions existent mais soit dans une forme virtuelle (elle existe comme une virtualité qui sera réalisée dans le futur) soit dans une forme potentialisée, c'est-à-dire elle est là mais retardée : c'est le "Ça prendra du temps » toujours invoqué dans le discours politique pour justifier des promesses qui ne sont pas tenues ou des actions jetées aux oubliettes, ou encore les projets vidés de leur contenu en les rendant inutiles et inefficaces, sans la 
valeur qui les définissait, dévitalisation réalisée par une sorte d'opération d'atténuation. Cette opération ne signifie pas la disparition de l'action ou encore sa substitution par une autre, mais tout simplement sa décadence, son déclin, avec des effets de pérennisation des situations, et avec le maintien de l'état « entre-deux », un état où l'acteur politique n'est ni conjoint ni disjoint de l'objet ou de son action, lesquels, comme on le sait, demandent d'attendre, exigent de la patience (Fontanille \& Zilberberg, 1998, pp. 91-111).

L'autre forme sous laquelle la transformation peut être appréhendée est celle du résultat, en tant que produit d'une action. Du point de vue aspectuel, cette forme correspond à la valeur perfective, donc accomplie, complétement réalisée, ou, si l'on veut, "menée à son terme ». Cette interprétation de la transformation met l'accent sur la démarcation, c'est-à-dire sur la distinction claire et nette entre un moment initial et un moment final qui se distinguent précisément, l'état final ne conservant aucune valeur, aucun vestige de l'état initial. Entre eux, il s'agit d'introduire des grands intervalles, des différences sans appel et des limites parfaitement perceptibles. Au lieu d'une politique du potentiel, du germe et de la semence, on oppose une politique de l'impatience et de l'immédiateté. Cette politique peut s'exprimer à travers deux modes d'existence ou de présence différents :

a) d'un côté il y a le mode d'existence réalisant qui exige qu'on accomplisse une action et qu'on passe à autre chose et ainsi de suite dans une sorte de concentration maximale sur chaque sujet : je prends l'exemple des propos que l'ancienne candidate socialiste aux élections présidentielles de 2007 en France, Ségolène Royale, avait tenus lors d'un entretien donné au journal Le Monde : «Dans mon livre, je cite Roosevelt, qui explique à quel point ses cent premiers jours ont été déterminants pour la suite. Là, il aurait fallu aller beaucoup plus vite : le mariage pour tous aurait dû être fait l'été dernier, de même que la réforme sur la décentralisation. Il fallait réformer par ordonnances dès le début » (Le Monde, 13 mai 2013); 
b) d'un autre côté, la construction d'un mode d'existence actualisant, de «mobilisation » pourrait-on dire, avec la création permanente de manques à combler. Dans ces cas, c'est l'acteur politique lui-même qui convoque les objets de l'action à réaliser et désigne leur valeur ouvrant le politique vers un futur à rejoindre créant des expectatives qui doivent constamment être renouvelées et où chaque action et chaque projet politique chassent ceux d'avant en en faisant émerger des nouveaux. La différence avec la modalité précédente demeure dans le fait que les valeurs et les projets de ce genre ne sont pas nécessairement réalisés : on construit le manque, on indique un chantier politique, et puis on passe à une autre chose et on en invente une autre qui à son tour restera à cet état actualisé mais non réalisé. Cette conception de la politique comme discours et comme pratique - a le mérite de poser des questions et de pointer des objets politiques mais elle risque de se limiter à n'être qu'un identificateur, voire un créateur des problèmes politiques qu'il révèle, voire qu'il invente. Il s'agit de la politique de l'éclat, des effets d'annonce, de celle dont on accusait l'ancien président de la République française Nicolas Sarkozy, à qui on reprochait de ne faire que des propositions de projets vite abandonnées ou de soulever des polémiques et des problèmes sans y remédier ni y répondre ; d'autres diront que cela avait au moins le mérite de dévoiler les problèmes souvent occultés ou tabous.

Dans les deux cas, l'effet induit est similaire pour ce qui concerne le tempo de la narration et de la transformation, rythmé et vif, n'ayant pas le temps de traîner et avançant d'événement en événement. Que l'action soit complétement réalisée ou simplement actualisée, elle est toujours dépassée, car son destin est de laisser sa place à une autre avec ce que cela comporte d'exaltation et d'agitation pour le sujet politique qui est en permanence « sur le vif » sans « laisser du temps au temps », en particulier dans le mode d'existence actualisé dans lequel il n'y a pas de temps pour que l'action ou le discours politique arrivent à leur terme.

On voit bien que l'horizon stratégique que ces deux modalités de la transformation dessinent est défini par un schéma de l'agir politique à deux directions possibles déterminées par la relation entre, d'un côté, l' « intensité » et la « densité », la « force » et l'incidence de la valeur et du sens du discours et/ou de la pratique politiques et, de l'autre côté, 
par l'«étendue », la « diffusion » et le champ « d'application » de ces mêmes discours et pratiques.

Ce schéma fait apparaître deux conceptions de l'action politique et de son efficience en tant que transformation des sociétés qui coïncident avec les deux grandes visions du changement social et culturel :

a) d'un côté une vision de l'action politique comme renversement, intense, qui peut être concentrée dans le temps et dans l'espace d'influence et qui, on suppose, finira par traîner le reste du social, comme synecdoque, exempla et/ou autoritas, ou qui peut être généralisée en impliquant toute la société au même moment, comme dans les révolutions ;

b) de l'autre côté, une vision du changement de la société par diffusion des valeurs et idées, par porosité et influence, donc une idée de la transformation sociale qui se réalise à travers le changement graduel et continu, par transition continue et, si l'on veut, imperceptible, silencieuse, par propagation, par tendance : ce sont ces dites « batailles d'idées » qui doucement mais sans interruption finissent par exemple par modifier un état d'opinion, comme quand on parle de la « banalisation des idées du Front National » ou comme dans le cas de la lente mais progressive et inéluctable acceptation par les sociétés occidentales du triomphe sans partage du libéralisme comme doctrine sociale et économique. Le concept d'« hégémonie culturelle» forgé parl'activiste et théoricien communiste italien Antonio Gramsci comme modèle de transformation sociale et politique (ou de son maintien) recouvre parfaitement cette modalité extensive et graduelle évoquée. Pour construire une « hégémonie culturelle » contraire à l'hégémonie bourgeoise dominante qui permette un renversement final de la société, il faut, toujours selon Gramsci, avant toute tentative d'une " guerre de mouvement », faire une « guerre de positions » qui permettra de réaliser un long travail idéologique, une lente préparation du terrain au sein de la société civile pour installer les valeurs qui permettront par la suite de prendre et de conserver le pouvoir ; toujours selon les mots de Gramsci : «La formation d'une conscience collective unitaire demande des initiatives et des conditions multiples. La diffusion d'un mode de penser et d'agir homogène » (Gramsci, 1971). 
Dans ces différentes formes de la transformation on voit aussi émerger des régimes narratifs différents. D'une part, l'organisation narrative issue de la description de l'univers du conte merveilleux faite par Propp autour des épreuves - qualifiante, décisive et glorifiante qui constitue le socle du modèle narratif de la sémiotique et qui privilégie une vision téléologique du parcours du récit, lequel doit nécessairement se terminer par la restauration d'un ordre et par la reconnaissance et la rétribution du héros ou par la condamnation en cas de non réalisation ou d'échec, cette sanction étant la condition même pour l'existence du sujet et pour l'attribution du sens au monde. Celleci est la forme syntagmatique qui correspondrait au premier modèle de la transformation sociale que nous avons construit. D'autre part et parallèlement à cet univers de l'action " accomplie " (de manière positive ou négative), un autre monde corrélatif se dessine. À côté donc de deux régimes narratifs, celui de l'émergence de nouvelles valeurs ou de nouveaux scénarios politiques et celui d'un agir produisant la disparition d'anciennes valeurs et états de discours, un autre régime fait son apparition, celui qui n'exige pas de transformations catégoriques. Ce régime combine sans problème les deux antérieurs : celui de la disparition et celui de l'émergence des valeurs, en faisant cohabiter des valeurs et des actions contradictoires car tout dans l'action est gradué, imperfectif, et non catégorique et perfectif. Ainsi donc, si l'on prend comme exemples les discours et les pratiques à l'issue des élections politiques, on verra qu'il y a des pratiques et des discours où l'on démontrera que l'on peut triompher et échouer en même temps et construire les étranges et pourtant réelles figures du perdant/gagnant ou, inversement, du gagnant/perdant : pensons à la bizarrerie des résultats des élections italiennes de 2013 où le perdant (Silvio Berlusconi) a été le gagnant et où le gagnant (le dirigeant du Parti Démocratique italien, Pier Luigi Bersani) le perdant.

Finalement nous retrouvons tout le mystère et le charme de la mythologie en politique : l'heureux mariage des contraires, le triomphe de l'oxymore, ce qui sans discussion est vraiment et malgré tout inénarrable. 


\section{Références}

Fontanille, J., \& Zilberberg, C. (1998). Tension et signification. Sprimont : Mardaga. Gramsci, A. (1971). Lettres de prison. Paris : Gallimard.

Landowski, E. (2004). Passions sans nom. Paris : PUF.

Marrone G. (2010). « Brand on the run ». Uno sguardo semiotico su Slow Food. Lid'O: lingua italiana d'oggi, 8, 271-291.

Ricœur, P. (1983). Temps et récit. 1. L'intrigue et le récit historique. Paris : Seuil.

Rosa, H. (2010). Accélération : une critique sociale du temps. Paris : La Découverte.

Salmon, C. (2007). Storytelling. La machine à fabriquer des histoires et à formater les esprits. Paris : La Découverte.

Zilberberg, C. (2004). Éloge de la concession. Disponible à http://www.claudezilberberg. net/pdfs/Concession.pdf. 